\title{
Exemplar Based Recognition of Visual Shapes
}

\author{
Søren I. Olsen \\ Department of Computer Science, \\ University of Copenhagen, Denmark
}

\begin{abstract}
This paper presents an approach of visual shape recognition based on exemplars of attributed keypoints. Training is performed by storing exemplars of keypoints detected in labeled training images. Recognition is made by keypoint matching and voting according to the labels for the matched keypoints. The matching is insensitive to rotations, limited scalings and small deformations. The recognition is robust to noise, background clutter and partial occlusion. Recognition is possible from few training images and improve with the number of training images.
\end{abstract}

\section{Introduction}

Several recent successful approaches to shape recognition $3,5,6,8,9,9$ are based on attributed image keypoints. By choosing the descriptors carefully, such approaches has shown robust to rotations, scalings, illumination changes, 3D camera viewpoint including minor deformations, and - probably most important background clutter and partial occlusion. The present approach follows this line of research. In the recognition phase the focus is not on instance detection, but on semantic contents report. The semantic contents of a training image is supplied as a list of names (labels), e.g. 'house', 'chair' etc. The set of names form the vocabulary by which test images can be recognized. Given a new unlabeled images the system reports the label(s) with strongest support from the matched keypoints. Also an image of the recognized structures is produced.

\section{Previous Work}

Probably the most influential early work using keypoints for recognition is the paper of Schmid and Mohr 9]. Here keypoints are detected at multiple scale levels using a Harris corner detector, and a vector of differential invariants is used as descriptor. Recognition is done using multidimensional indexing and voting and by applying a model of the shape configuration, i.e. the spatial relationship between the keypoints defining a model. In later work [10, 6] the use of different interest point operators has been evaluated, and the Harris detector has been combined with other approaches to result in a scale and affine invariant detector.

Another influential work is the papers by Lowe [3, 4, 5. Here the keypoints are detected in scale-space as the local extremes in the convolution of the image 
with the difference of Gaussians. The descriptor is chosen by sampling the image gradient orientation in a rectangular grid centered at the keypoint and aligned with the dominating local gradient orientation. By using a variant of the $\mathrm{k}-\mathrm{d}$ tree for indexing, input keypoint descriptors are matched to their most similar neighbor in the database of trained descriptors. Then sets of 3 matched keypoints defining the pose are grouped using the Hough transform [5]. Next, an accurate pose of the recognized object is fitted by an iterated least squares affine fit with outlier removal. Decision to reject or accept the model hypothesis is finally made based on a probabilistic model [4].

The present work may be seen as a refinement of [8]. In this work keypoints are detected in scale-space using a model of end-stopped cells [2] and using centers of circular contours. The former type of keypoint identify corners (2 legs), junctions (3 legs) and more complicated structures with 4 legs. The directions of the legs is well suited for indexing. In the present approach the keypoint types are unchanged, but the detection method has been improved. Compared with the keypoint types used in [5, 6, fewer keypoints are in general detected. These are larger scale features more likely to mark positions with high semantic contents.

In [8 a 2-dimensional histogram of local edge point gradient orientation located within an angular sector relative to the keypoint is used as descriptor. Comparison between an input and a database keypoint is made by a modified $\chi^{2}$-test. Due to quantization problems this descriptor often does not perform well. To achieve a recognition invariant to rotations, scalings act. one method is to choose descriptors that are invariant to such transforms [9, 6]. This approach is reasonable only if the transformations can model the expected deformations well. In the present work this is not appropriate, because the chosen descriptor is not very local. To achieve rotational invariance the descriptor measurements may be made in a coordinate system aligned with the dominating local image gradient orientation [5. For the keypoints chosen in the present system there will be either several or no such orientations. In [5] the problem of multiple dominating orientations is solved by storing as many descriptors as there are such orientations. This reduces the time for single descriptor comparisons but increases the size of the database significantly. We take a third approach, using a descriptor that is not invariant to the transforms mentioned. Instead rotational invariance and insensitivity to minor deformations is left to the matching process. This choices will lower the size of the database at the expense of a larger computational complexity during the matching.

The present work is focused on reporting the semantic contents of an image in terms of one or several labels introduced during training. This is different from detecting the existence and pose of a specific object in an image [5, 9]. Loosely speaking, the method is aimed at object shape categorization rather than image-to-image matching. Thus a requirement of positional consistency between a group of neighboring query and database keypoints is not necessary. In the present system there is no spatial relations between keypoints defining a shape. The lost discriminative power is regained by choosing a descriptor with a larger spatial support. Each query keypoint may be matched to multiple database 
keypoints. The classification is then made using a simple voting mechanism where each match votes on the database keypoint label with a strength determined by the similarity between the two descriptors.

\section{Keypoint Detection}

First the essential aspects of the visual shape such as object outline contours and texture contours are extracted by edge detection using Gaussian derivative convolution and spatial non-maximum suppression of the gradient magnitude. The edge points are then used to detect the two types of keypoints: Circular structures and junctions (including corners). These keypoints mark image positions where the semantic content of the local image shape is locally rich. The core of the detection method is described in [8. Experiments however have shown that for junctions neither the estimated localization nor the number and directions of the legs defining the junction are sufficiently stable or accurate.

In the present work each detected junction is validated in a refinement step. For each detected junction the edge points in the local neighborhood are grouped according to their local angular positioning and gradient orientation. Then a straight line is fitted to the points in each group. If few points contribute to the fit, if the residual of the fit is bad, or if the distance from the junction point to the fitted line is large, then the group is discarded. If less than 2 groups remain the junction is discarded. Otherwise the fitted lines are intersected using least squares. If the residual of the fit is large or if the intersection point is far from the initial position of the junction this is discarded. Otherwise the intersection point becomes the new position of the junction. Thus, the remaining keypoints are well localized and defined by locally straight edges with a well defined orientation.

To achieve an optimal detection of keypoints previous methods have used a multi-resolution (scale-space) approach where keypoints initially are detected at a set of scale levels, then linked through scale-space, and accepted only if some strength measure is extreme. In [6] a single optimal scale for each keypoint is found. In [5] several (extremal) keypoints may be selected. Experiments have shown that both corners and junctions may be inconsistently detected over scale, i.e. the number of legs and their orientation may differ. In the present approach a scale-space approach is used - not to ensure optimal detection - but to enable recognition of shapes scaled in size and to prune unstable detections. First keypoints are detected in scale-space, using a sampling of $\sqrt[4]{2}$ corresponding to 3 samples per octave, and grouped in scale-space according to their type and positioning. For each group the dominating directions of the legs are found by histogram analysis. Then a number of the registered representations that are consistent with respect to the number and directions of the keypoint legs are selected by sampling. The sampling is made such that the scale distance between two selected items is at least 2 scale levels. Finally, all isolated non-selected keypoints (with no other selected keypoints in their neighborhood spatially as well as w.r.t. scale) is selected as well. This guarantees that only stable or unique representatives are chosen. The sampling is chosen as a compromise between a 
small amount of redundancy among the representatives and a good coverage of different neighborhood sizes coded by the descriptors. The net result is a reduction in the number of selected keypoints (often by $50 \%$ ) and that most of the selected ones are supported by similar detections at other scale levels. Especially for junctions, most spurious leg detections are removed in this process.

\section{Keypoint Descriptors}

In the present work the choice of descriptors to a large extend follows the approach of local gradient sampling proposed by Lowe [3,5]. However, both the sampling points, the quantization, and the descriptor matching is different. The exemplar approach, relying solely on the statistics of isolated image patch matches, requires that the keypoint descriptor is chosen to code the shape in a sufficiently large image patch in order to possess sufficient discriminative power. However it also must not be too selective. A reasonable trade off between discriminative power and generalization is experimentally found to correspond to a support radius between 10 and 20 pixels. In [5] a rectangular sampling grid is used. This grid is aligned with the dominating direction of gradient orientation. If several peaks in the histogram of gradient orientation are present, several keypoints are generated. This will double/triple the size of the database for corners/junctions. The advantage of the redundancy is a simpler matching of descriptors to the database. The disadvantage is that more descriptors should be matched to a larger base. We choose to represent each keypoint only once and to pay the price of having to "rotate" the descriptor vector for a each descriptor comparison. For this choice a sampling in a rectangular grid is inappropriate. We choose to sample the gradient information in 5 rings with radii about $3 i, i=1 . .5$, and with a sampling distance of about 3 pixels in each ring, corresponding to 6 , $12,18,24$ and 30 samples (90 in total). The segmenting of the disc is made using the k-means algorithm. Each pixel within a distance of about 18 pixels from the keypoint is classified to the nearest segment sample point and the gradient with largest magnitude within each segment is chosen (see Figure 1). We then code the gradient magnitude and the angle between the gradient orientation and segment direction. Each of the two segment component are quantized to 4 bits
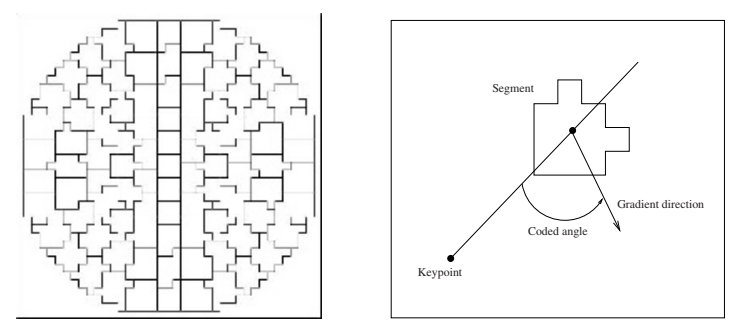

Fig. 1. Sampling regions (left), and coded angle for a segment (right) 
each. Thus the description vector has the size of 90 bytes. The coding of the individual sample elements is invariant to rotations, but their position in the descriptor vector is not.

The motivation for inclusion of the (quantized) gradient magnitude in the descriptor is that this information allows a (more or less pleasing) reconstruction of a coded image. Also, a "mental image" of the recognized structures in a query image can be made based on the stored data, not the query data. As an example Figure 2 shows a query image, an image reconstructed from the coded data, and two reconstructions based on matched database descriptors. The database was constructed using 89 objects from the COIL-100 database [7], each object seen from 8 different viewing angles (45 degree separation in depth). The two query images differed from the nearest training image by a rotation of 10 degree (in depth).

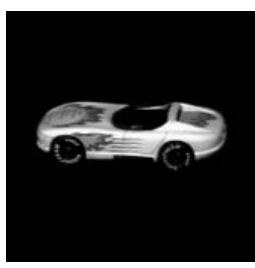

(a)

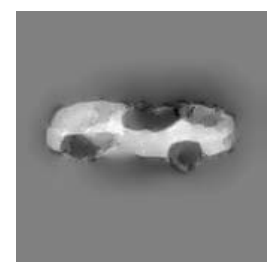

(b)

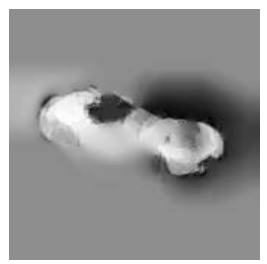

(c)

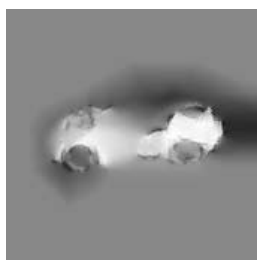

(d)

Fig. 2. Image (a) and reconstruction (b) based on the 63 detected keypoints in the image. Two reconstructions (c) and (d) based on 22 and 14 keypoints

The reconstruction is made coarse to fine. The solution at a coarser level is used as initial value in the reconstruction at the next finer level. At each level the reconstruction is made in three steps. First a map of gradient magnitudes is constructed from the database descriptors at the positions defined by the matching input keypoints. Only matches to the winning label is used. Next, this map is anisotropically smoothed and the ridges detected. Ideally, these correspond to the recognized shape contours. For each point on the detected ridge the coded gradient information defines two equations in the partial derivatives of the reconstructed surface. Using standard regularization, a simple iterative updating procedure is finally applied. For simplicity a fixed number of iterations is used. Since no absolute intensity values are known the reconstruction can be made up to a additive constant only. In areas with few keypoints the reconstruction will be poor. Areas with no recognized keypoints will be reconstructed by a constant initial value of middle-gray. Thus the reconstructed image probably will be of low quality, but nevertheless show the recognized structures. 


\section{Shape Recognition}

The database of stored keypoints is organized in four groups containing circular structures, corners, junctions, and structures with more that 3 legs. Corners are indexed by the quantized angle between the two legs. Junctions are accessed using a 2-dimensional array indexed by a similar quantization of the angles between the first and the other two legs. The stored keypoint descriptions for circular structures and structures with more than 3 legs are relatively few and compared to the query keypoints through an exhaustive search. To handle large rotations and angular quantization errors several bins are checked for corners and junctions. For an $n$-legged keypoint $n \cdot 2^{n-1}$ bins are checked corresponding to the $n$ possible ways one input leg can be matched to the first leg of the stored keypoint and the $2^{n-1}$ combinations the $n-1$ angles can be quantized to the two nearest integer values. Experiments show that the indexing step reduce the number of further comparisons by a factor of 5-20. Next each input keypoint is compared to the stored representations in the union of checked bins. In [8] a fast comparison using a measure of asymmetry was used to further limit the computational burden. Such fast tests are still relevant but are - for simplicity omitted here.

A query keypoint is compared to a database keypoint by comparing the descriptors of gradient sample values. First the query descriptor is rotated to make the orientation of the first legs of the keypoints match. To eliminate quantization problems three rotation angles, corresponding the nearest three integral sampling numbers, is determined for each ring in the descriptor. Based on a score value the best of the three rotations is chosen independently for each sample in each ring, and a total match score is computed as a weighted sum over the 5 rings. This procedure ensures rotational invariance and that a significant amount of non-trivial deformation can be handled. The weights are chosen inversely proportional to the number of samples in each ring, i.e. inversely proportional to the sample distance. Within each ring the score is computed as a sum of gradient sample differences. Let $\mathbf{g}^{q}=\left(v^{q}, m^{q}\right)$ and $\mathbf{g}^{d b}=\left(v^{d b}, m^{d b}\right)$ be the quantized sample values of orientation and magnitude for a query and a database segment, and let $d m=\left|m^{q}-m^{d b}\right| / 16$ and $d v=\left|v^{q}-v^{d b}\right|$, where the value 16 corresponds to the number of magnitude sampling intervals. Then the gradient sample difference is defined by:

$$
\operatorname{dist}\left(\mathbf{g}^{q}, \mathbf{g}^{d b}\right)= \begin{cases}d m \cdot(d v+1) & \text { if } d v<2 \text { and } d m<0.5 \\ 1 & \text { otherwise }\end{cases}
$$

Thus small gradient orientation differences are punished mildly, and larger differences equally hard. Finally, the match score is converted to a value $\in[0: 1]$ with 1 corresponding to a perfect match. The match is accepted if this value is above an empirically determined threshold.

Each query keypoint may be linked to zero, one or a few database keypoints, each link attributed with a match-score. Since in the present study we want to report the semantic contents as specified by the list of training names, a simple voting procedure is applied. Each match votes with its score as strength and has 
as many votes as there are names in the list associated with the matched database elements. Then the list of names are sorted according to the accumulated score value and the top-ranking name selected.

For the matches contributing to the highest ranking semantic association, a confidence $C$ of the naming is estimated. This is based on the total support score $S_{0}$ for the most likely naming and computed by: $C=\left(S_{0} / \sum S_{i}\right) \times\left(1-\exp \left(\frac{s_{0}^{2}}{2 \sigma^{2}}\right)\right)$. Thus $C$ will be low if the naming is not unique or if the support score for the naming is not strong.

When training the system with a new image, a list of names is supplied. A keypoint in a new training image is installed if it cannot be matched to any previously seen keypoint. If the keypoint can be matched to a database keypoint, the list of labels for the database keypoint is extended with the new names. The description vector of the database exemplar keypoint is not updated in any way.

\section{Experiments}

In the experiments reported below the system was first fed with a number of images, each annotated with one label. Then the system was tested by presenting it to new unnamed images and the results of the classification was monitored. In most of the reported experiments the COIL-100-database [7 was used. This contains 100 objects each imaged from a full circle of 72 view positions. Prior to the experiments the images were preprocessed to correct for a 2-pixel column warp-around error, an incorrect constant background value and noise level, and added a supplementary border of 20 pixel width to enable detection of keypoints near the old image border. Also, the images were converted to gray-scale.

First the performance on the COIL-100-database was tested with respect to the number of objects. For subsets of the 100 objects, 8 views with an angular separation of 45 degrees were used for training and the remaining 64 views for test. First the system was first tested on all 100 objects. Then, approximately 10 objects that had the worst classification results were iteratively removed, and the system was retrained and retested on the smaller database. This procedure results in an optimal assessment curve. In all runs the threshold on the confidence $C$ was zero, implying that all images were classified. Below in Figure 3 the results are summarized. As expected the performance decreases as the number of objects increases. For less than approximately 50 objects the misclassification rate was below $4 \%$.

Next, the ability of the system to perform well, when trained on few training images, were tested on subsets of the 37 object images of the COIL-100 database. The test also shows the ability to recognize objects rotated in depth. The ratio of training images to test images was varied from 36/36 to 4/68 corresponding to an angular interval between the training image from 10 to 90 degrees. As before, a confidence level of zero was used. Figure 4 below shows that a misclassification rate below $4 \%$ is possible with as few as 6 training images per object corresponding to an angular separation of 60 degrees. Analysis showed that the average confidence value for correct and false classifications was 0.51 and 
0.35 with standard deviations 0.08 and 0.06 . The two distributions are highly overlapping making a threshold-based separation difficult. This was typical for other experiments as well. Assuming normal distributions an optimal confidence threshold of 0.32 was found. Using this value will equal the number of accepted false classifications and the number of rejected true classifications. Figure 4 also shows the misclassification rate and the rate of unclassified images for $C=0.32$.

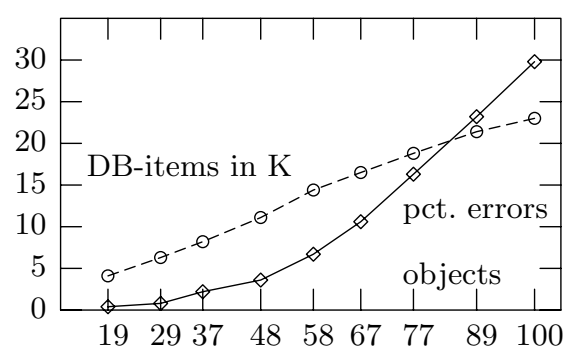

Fig. 3. Misclassification rate on subsets of the COIL-100 database, and the size of the build databases in kilo keypoints

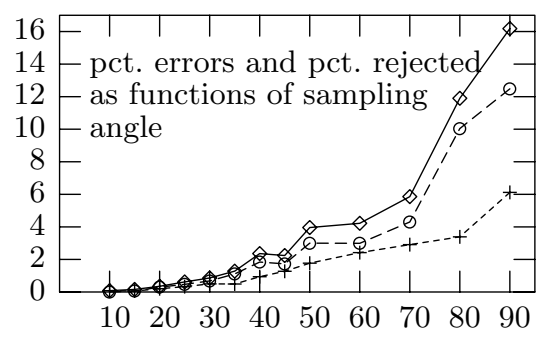

Fig. 4. Misclassification rate for different number of training images using $C=0$ (upper) and $C=0.32$ (middle), and the percentage of unclassified images for $C=0.32$ (lower)

Rotation in the image plane was tested similarly. For subsets of zero-angle images of the COIL-100 database used for training, the system was tested on 71 synthetically rotated versions of each training image using a rotation step of 5 degree. Figure 5 shows that the misclassification rate was low when less that about 45 objects were to be recognized, and that the misclassification rate increased smoothly until the break-down at about 85 objects. For a small number of objects the recognition rate was independent of the rotation angle. For large databases the recognition rate was slightly better for images rotated approximately a multiplum of 90 degrees. Misclassification may happen when several

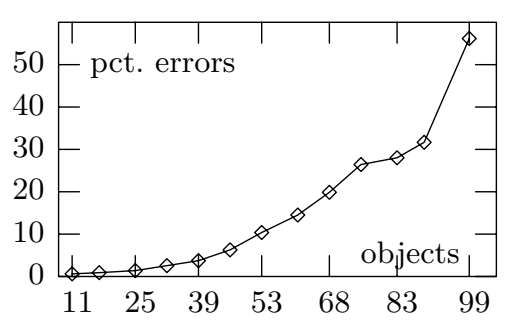

Fig. 5. Misclassification rate for images rotated in the image plane as a function of the number of training images

\begin{tabular}{|c|c|c|}
\hline ranking & 37 objects & 89 objects \\
\hline 1 & 97.8 & 76.9 \\
\hline $1-2$ & 99.1 & 83.4 \\
\hline $1-3$ & 99.4 & 86.8 \\
\hline $1-4$ & 99.7 & 88.5 \\
\hline $1-5$ & 99.7 & 89.8 \\
\hline $1-6$ & 99.8 & 90.9 \\
\hline $1-7$ & 99.9 & 91.8 \\
\hline $1-8$ & 99.9 & 92.5 \\
\hline $1-9$ & 99.9 & 93.1 \\
\hline $1-10$ & 99.9 & 93.7 \\
\hline
\end{tabular}

Fig. 6. Accumulated histograms of the recognition rate for the top-10 ranking of the correct classification 
object share substructures making them alike from certain view angles. In such cases, and when the queries are expected to show several objects, a list of the top-ranking classifications may be useful. For two subsets of objects of the COIL100 database, the system was trained on 8 images with 45 degree separation, and tested on the remaining images. Then an accumulated histogram of the ranking of the correct classification was constructed. As shown in Figure 6 the correct naming was in most cases among the first few elements in the classification priority list. However, for the larger object subset still many queries seems difficult to classify correctly. One reason is that for these subsets several of the objects were very alike. Another reason is that object names trained from images with many keypoints tend to receive more votes than object names trained from images with few keypoints. Thus images of simple objects may be misclassified. This is due to the simple unnormalized voting scheme. Please note that in general neither normalization with respect to the number of keypoints in each training images nor to the number of identically labeled keypoints seems reasonable. The first choice will penalize shapes trained from images also showing background clutter. The second choice will penalize shapes with large variability. Experiments showed that in general neither type of normalization improved the performance.

Finally, the database build on 8 views of each of 37 objects from the COIL100 collection was extended with training images of cars in natural (mostly urban) scenes. The latter images as well as the test images were selected from the car database [1]. The training images had a size of $40 \times 100$ pixels. The 144 query images were much larger and showed a significant amount of background clutter and partial occlusion. Figure 7 shows the amount of misclassification as a function of the number of car images in the training set. For less than 32 training images of cars the recognition rate was poor. This is not surprising because of the difficulty of the images and because the cars may point both left and right and may be lighter or darker than the background (corresponding to 4 different types of cars). For larger training sets the misclassification rate stays constant at a level about $3 \%$. This is caused by $4-5$ images with heavy background clutter coincidentally giving rise to keypoints matching better to some of the distractors (keypoints from the COIL-object-views).

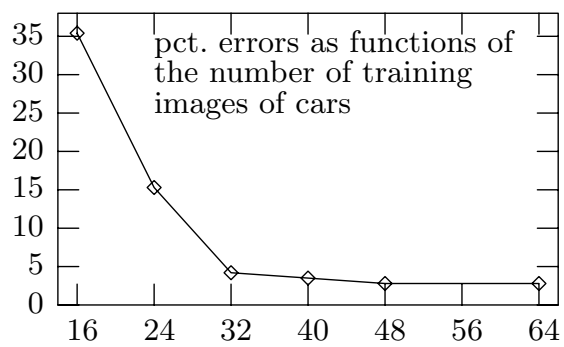

Fig. 7. Misclassification rate on images of cars in natural scenes as a function of the number of training images of cars 


\section{Conclusion}

An exemplar based recognition scheme using attributed keypoints has been described and a few preliminary experiments has been reported. The results indicate that the system is robust to rotations, limited scalings, noise, small deformations, background clutter, and partial visibility, when the number of objects are limited (e.g. $<50$ ). The stability w.r.t. rotations in depth has been shown to be good, and it has been shown that recognition is possible based on few training images. The results show that good performance is achievable using only local information for keypoint matching. Schmid [9] reports an experiment with 20 objects from the COIL collection, using a 20 degree separation between training as well as test images. We achieve a similar recognition rate of 99.6, but using less than half the number of training images. Much computational effort has been put on the initial keypoint detection leaving fewer - but hopefully more stable and semantically rich - keypoints. It is left for future research to investigate whether this approach is advantageous with respect to the matching success. Automatic learning of significant keypoints as opposed to keypoints caused by background clutter and irrelevant details is of high importance for achieving a good recognition rate and to avoid the database being filled with useless data. In the present approach - having no concept of an object - this might be done by removing rarely used keypoints. The viability of this approach is left for future research.

\section{References}

1. S. Agarwal, A. Awan, D. Roth: UIUC Image Database for Car Detection; http://12r.cs.uiuc.edu/ cogcomp/Data/Car/

2. F. Heitger, L. Rosenthaler, R. Von der Heydt, E. Peterhans, O. Kubler: Simulation of Neural Contour Mechanisms: from Simple to End-stopped Cells, Vision Research vol. 32, no. 5, 1992, pp. 963-981

3. D. Lowe: Object Recognition from Local Scale-Invariant Features, Proc. 7'th ICCV, 1999, pp. 1150-1157

4. D. Lowe: Local feature view clustering for $3 D$ object recognition, IEEE Conf. on Computer Vision and Pattern Recognition, Hawaii, 2001, pp. 682-688

5. D. Lowe: Distinctive Image Features from Scale-Invariant Keypoints Int. Jour. of Computer Vision 60(2), 2004, pp. 91-110

6. K. Mikolajczyk, C. Schmid: Scale $\&$ Affine Invariant Interest Point Detectors Int. Jour. of Computer Vision 60(1), 2004, pp.63-86

7. S.A. Nene, S.K. Nayar, H. Murase: Columbia Object Image Library, 1996; http://www1.cs.columbia.edu/CAVE/research/softlib/coil-100.html

8. S.I. Olsen: End-Stop Exemplar based Recognition, Proceedings of the 13th Scandinavian Conference on Image Analysis, 2003, pp. 43-50.

9. C. Schmid, R. Mohr: Local Grayvalue Invariants for Image Retrieval, IEEE trans. PAMI, 19(5), 1997, pp.530-535

10. C. Schmid, R. Mohr, C. Bauckhage: Evaluation of Interest Point Detectors, Int. Jour. of Computer Vision 37(2), 2000, pp. 151-172 